\title{
ESTUDO CLÍNICO-EPIDEMIOLÓGICO DE PACIENTES COM EPILEPSIA MIOCLÔNICA JUVENIL EM SANTA CATARINA
}

\author{
ROBERTO FIGUEREDO*, PAULO CÉSAR TREVISOL-BITTENCOURT**, \\ JULIANO BALSINI DE MOURA FERRO***
}

\begin{abstract}
RESUMO - Realizamos estudo retrospectivo em 26 pacientes com epilepsia mioclônica juvenil (EMJ) no intuito de traçar seu perfil clínico e estabelecer a prevalência da síndrome entre pacientes com epilepsias diversas. Critérios de inclusão no estudo foram: a) abalos mioclônicos, preferentemente matinais; b) início dos sintomas entre 8 e 26 anos; c) laudo eletrencefalográfico típico; e d) boa resposta ao valproato de sódio (VPA). A prevalência de EMJ, entre 939 pacientes com epilepsias diversas, foi de 2,8\%. Observou-se nítido predomínio do sexo feminino $(73,1 \%)$. A idade de início dos sintomas variou de 7 a 18, média de 13 anos. Crises tônico-clônicas e de ausência eram apresentadas concomitantemente por, respectivamente, 92,3 e 19,2\% da amostra. Além disso $92.3 \%$ deles apresentavam atividade epileptiforme típica em seus eletrencefalogramas e 76,9\% evidenciaram boa resposta ao VPA com dose diária variando de $500 \mathrm{mg}$ a $1500 \mathrm{mg}$.
\end{abstract}

PALAVRAS-CHAVE: epilepsia, mioclonias, valproato de sódio.

\section{Clinical-epidemiological study of patients with juvenile myoclonic epilepsy in Santa Catarina State -Brazil}

ABSTRACT - We aimed to characterize the clinical profile and to establish the prevalence of juvenile myoclonic epilepsy (JME) among 939 consecutive patients referred to our epilepsy clinic. Inclusion criteria in the study were: a) myoclonic jerks, preferably on awakening; b) beginning of the symptoms between 8 and 26 year-ofage; c) typical pattern of electroencephalographic discharges; and d) good response to sodium valproate (VPA). In a retrospective design, 26 cases of JME were identified (prevalence 2.8\%). Most of these patients were female (73.1\%). Mean age at onset of symptoms was 13 (range 7-18). Tonic-clonic seizures were reported in $92.3 \%$ and absence seizures in $19.2 \%$. Besides, $92.3 \%$ of the patients had typical EEG epileptiform activity and $76.9 \%$ had good response to VPA with daily dose ranging from $500 \mathrm{mg}$ to $1500 \mathrm{mg}$.

KEY WORDS: epilepsy, myoclonic jerks, sodium valproate.

Epilepsia mioclônica juvenil (EMJ) é síndrome relativamente frequente, representando cerca de 4 a $12 \%$ de todos os pacientes com epilepsia ${ }^{1}$. Ambos os sexos são afetados mas não podemos afirmar se o são na mesma proporção ou se há predomínio de um sobre o outro². A idade de início das manifestações varia entre 9 e 27 com maior acometimento na faixa de 13-20 anos ${ }^{3}$. A tríade característica consiste de crises de ausência típicas, abalos mioclônicos ao despertar e crises tônico-clônicas, frequentemente precedidas por abalos. As crises de ausência na EMJ costumam iniciar entre 5 e 16 anos mas têm participação restrita, pois estão presentes entre 10 a $39 \%$ dos pacientes. ${ }^{1,3}$ Abalos mioclônicos estão presentes em $100 \%$ dos $\operatorname{casos}^{3}$ e costumam ocorrer entre 1 a 6 anos após manifestações das crises de ausência. Entretanto, naqueles que não iniciam com ausências, tais crises usualmente começam por volta dos 10 anos de idade. Por sua vez, as crises tônico-clônicas estão presentes em 90 a $95 \% 3$ dos pacientes e costumam surgir alguns meses após as primeiras manifestações mioclônicas.

Clínica Multidisciplinar de Epilepsia da Policlínica de Referência Regional I/SUS, Florianópolis (SC), Brasil: *MD; **MD, MSc, Professor de Neurologia da Universidade Federal de Santa Catarina (UFSC); ***MD. Aceite: 22-janeiro-1999.

Dr. Roberto Figueredo - Rua das Camélias 49 - 88.040-530 Florianópolis SC - Brasil. E-mail: delfigue @ zaz.com.br 
As mioclonias se expressam por abalos, de intensidade média a moderada dos membros superiores, ombros, pescoço e, menos frequentemente, membros inferiores e pálpebras. Costumam ocorrer ao despertar e são provocadas por privação do sono, stress emocional, consumo de álcool e exposição a estímulo luminoso. Em geral se apresentam sem alteração da consciência. O exame neurológico e o nível de inteligência são geralmente normais. O eletrencefalograma mostra ritmo de base normal e típicos complexos de espícula-onda e/ou multiespícula/onda generalizados, bilaterais e $\operatorname{síncronos}^{3-5}$. Os achados clínicos e eletrencefalográficos típicos associados a frequente ocorrência de EMJ e outras formas de epilepsia em parentes de probandos afetados sugerem que EMJ tem forte predisposição genética ${ }^{6-10}$.

Por outro lado, a resposta ao tratamento específico com valproato de sódio (VPA) é altamente positiva, controlando as crises na maioria dos pacientes. Todavia, ao contrário do observado em diversas outras síndromes epiléticas, EMJ tende a reaparecer com a supressão da droga anti-epilética (DAE), mesmo após vários anos de remissão. Em virtude dessa peculiaridade, é desaconselhável a interrupção de tratamento bem sucedido.

Nosso estudo procura traçar o perfil clínico dos indivíduos com EMJ bem como estabelecer a prevalência desta condição entre pacientes com epilepsias diversas. O estudo da amostra, segundo critérios clínicos e eletrencefalográficos, foi feito na Clínica de Epilepsia da Policlínica de Referência Regional do Estado de Santa Catarina. Esperamos, com isso, contribuir para um pronto reconhecimento dessa condição sui generis, poupando esses pacientes de procedimentos iatrogênicos.

\section{MÉTODO}

Realizamos estudo retrospectivo transversal descritivo em 26 pacientes que apresentavam critérios típicos de EMJ. Toda a amostra é seguida na Clínica Multidisciplinar de Epilepsia (CME) da Policlínica de Referência Regional I/ SUS, por neurologista especialista em epileptologia, entre agosto/1985 e dezembro/1997. Todos os pacientes tiveram revistas suas histórias clínicas e estudos eletrencefalográficos. Após estabelecido o diagnóstico, iniciou-se tratamento com VPA. Caso o paciente estivesse recebendo outra DAE, haveria substituição gradual dela mesma através de associação, tendo como objetivo final, a monoterapia com VPA. Quando não houvesse boa resposta ou presença de efeitos adversos intoleráveis, seria instituída uma das DAEs consideradas de segunda linha para a condição²: fenobarbital (PB), primidona (PRM) e clonazepan (CLZ).

Todos os 939 prontuários de pacientes com epilepsia foram avaliados e separados para análise individual tendo em vista os seguintes critérios: a) abalos mioclônicos, preferentemente matinais, b) início dos sintomas entre 8 e 26 anos; c) laudo eletrencefalográfico típico e d) boa resposta ao valproato de sódio. Cabe ressaltar que nessa amostra foram desconsiderados todos aqueles pacientes que apresentavam crises não genuinamente epilépticas.

\section{RESULTADOS}

Dentre os casos de epilepsia, ao todo 939, encontramos 26 pacientes com EMJ, representando $2,8 \%$ da amostra. Dezenove pacientes (73.1\%) eram do sexo feminino e 7 (26.9\%), do masculino. Sua idade variou de 12 a 49 anos (média 29). A idade de início dos sintomas variou de 7 a 18 anos (média 13). O tempo de doença foi de 5 a 43 anos (média 17).

Os 26 pacientes do estudo apresentaram crises mioclônicas. As crises de ausência e tônicoclônicas estavam presentes, respectivamente, em 5 (19,2\%) e 24 (92,3\%) deles. Um dos pacientes apresentou crises parciais e possuía laudo tomográfico sugestivo de neurocisticercose.

Quatorze $(53,8 \%)$ pacientes tinham antecedente familiar de epilepsia com 15 familiares acometidos: 2 pais, 4 irmãos, 5 primos, 2 tios e 2 avôs.

Vinte e cinco (96\%) pacientes possuíam estudo eletrencefalográfico. Vinte e quatro $(92,3 \%)$ apresentavam laudo típico para condição: complexos de espícula-onda e/ou multiespícula/onda generalizados. No total, havia 39 exames. Treze (50\%) pacientes possuíam mais de um sendo que 5 $(19,2 \%)$ deles apresentaram laudo normal após tratamento adequado. 
Vinte pacientes $(76,9 \%)$ tiveram redução no número ou remissão das crises usando VPA com dose variando de 500 a $1500 \mathrm{mg} /$ dia (média de $1000 \mathrm{mg} /$ dia). Entretanto, em seis deles $(23,1 \%$ ) seu uso foi descontinuado porque não houve boa resposta às crises e/ou os efeitos colaterais foram intoleráveis.

\section{DISCUSSÃO}

A EMJ é condição relativamente frequente em nosso meio, cuja prevalência entre os casos de epilepsia se encontra na faixa de 4 a $12 \% .{ }^{1}$ Em nosso estudo, dentre 939 pacientes com epilepsias diversas, 26 deles se enquadraram nos critérios clínicos de EMJ, perfazendo 2,8\% da amostra. É provável que as características da CME expliquem essa diferença. Ela atende fundamentalmente pacientes adultos e, desde que EMJ geralmente inicia na infância/puberdade, muitos destes pacientes provavelmente serão encontrados em clínicas de neuropediatria. Desta maneira, a cifra encontrada é uma estimativa mínima dos pacientes com esse problema em nossa sociedade.

Delgado-Escueta \& Bacsal $^{2}$ encontraram igual acometimento entre os sexos. Por outro lado, no estudo de Yacubian e $\mathrm{col}^{4}$ houve predomínio do sexo feminino. Nosso estudo também sugere isto, pois 19 dos pacientes acometidos eram do sexo feminino $(73,1 \%)$.

$\mathrm{Janz}^{3}$, em sua casuística, descreve idade de início dos sintomas entre 8 e 26 anos com predomínio entre 12 e 18 anos. Em estudo posterior ${ }^{5}$, com número de casos menor, a idade de início variou de 8 a 18 anos, média de 14 . Nossos dados são similares neste aspecto, com pacientes iniciando sintomatologia entre 7 e 18 anos, média de 13.

Todos os pacientes da nossa casuística apresentaram crises mioclônicas. Estas são habitualmente descritas pelos pacientes e familiares como "choques". As crises de ausência estão associadas à síndrome na faixa de 10 a $39 \%$ dos pacientes ${ }^{1-3}$. Em nossa amostra, o componente ausência esteve presente em cinco pacientes, correspondendo ao percentual de 19,2\%. Por outro lado, há incidência de 90 a $95 \%$ de crises tônico-clônicas nos pacientes acometidos ${ }^{5}$. Aqui também observamos cifra significativa: 24 pacientes (92,3\% deles), apresentaram crises tônico-clônicas. É importante salientar que, habitualmente, tanto as mioclonias como as crises de ausência são pouco valorizadas pelo paciente e seus familiares. De fato, usualmente há procura de assistência médica somente quando as crises tônico-clônicas estão presentes ${ }^{11}$. Mesmo assim, em alguns casos, os pacientes e seus familiares deixam de referir a marca registrada da síndrome, ou seja, as mioclonias ${ }^{12}$.

Um dos pacientes apresentava crises parciais com generalização secundária. Este possuía, por sinal, tomografia exibindo típicas lesões de neurocisticercose, sugerindo, dessa forma, a inusitada concomitância de duas síndromes epiléticos distintas. Esse fato para nós não foi de grande surpresa tendo em vista que a neurocisticercose é mal endêmico em várias regiões do país ${ }^{13}$.

Delgado-Escueta \& Bacsal $^{2}$ e Calderón-González e col. ${ }^{5}$ encontraram, respectivamente, $41 \%$ e $45 \%$ de pacientes com familiares acometidos. Nós encontramos 53,8\%. Em 14 pacientes havia antecedente familiar de epilepsia, com 15 parentes afetados: 4 irmãos; 2 entre os pais; 5 primos; 2 tios e; 2 entre os avós. Durner e col. ${ }^{8}$ sugerem que a síndrome esteja relacionada a marcadores do HLA no cromossomo 6. Não descartando componente genético, Whitehouse e col. ${ }^{10}$ não encontraram relação da condição a marcadores do HLA. Cabe ressaltar que em nossa região, quando vários membros de uma mesma família são epiléticos, devemos, em primeiro lugar, descartar o diagnóstico de neurocisticercose. Em nossa pesquisa não foi feito estudo epidemiológico com intuito de rastrear este tipo de paciente, representando, dessa forma, uma de suas limitações.

Com relação a achados eletrencefalográficos, Yacubian e col. ${ }^{4}$ verificaram atividade epileptiforme típica em $97.2 \%$ da amostra. Vinte e quatro pacientes, portanto $92.3 \%$ da nossa casuística, apresentaram típicos complexos espícula-onda e/ou multiespícula-onda generalizados. Cinco deles, após tratamento com VPA apresentaram laudo eletrencefalográfico normal. 
Há boa resposta à terapia com VPA em cerca de $80 \%$ dos $\operatorname{casos}^{2,3}$. Em nosso estudo, observamos que após introdução do VPA, 20 pacientes $(76,9 \%)$ apresentaram boa resposta com redução do número ou remissão das crises. Em seis pacientes $(23,1 \%)$, não houve boa resposta ora por efeitos colaterais intoleráveis, ora pelo não controle das crises. Estes, provavelmente, devido a má aderência ao tratamento. Os principais efeitos colaterais atribuídos ao VPA são: irritação gástrica; perda, adelgaçamento, mudança na cor e enrolamento dos pêlos; teratogenicidade (há aumento na incidência de espinha bífida); obesidade, sonolência, tremor ${ }^{14,15}$. Mais raramente, em pacientes idiossincrásicos, pode ocorrer hepatotoxicidade, com aumento das transaminases hepáticas e pancreatite ${ }^{14,15}$. Dentre os efeitos adversos possivelmente causados pela droga, em nosso estudo, foram citados: erupção cutânea, sialorréia e náuseas em um paciente alérgico ao medicamento; cefaléia (4 pacientes); queda de cabelos (4 pacientes); mudança na cor dos cabelos (1 paciente); sonolência (1 paciente) e sintomas psiquiátricos (1 paciente).

O moderno enfoque terapêutico em epilepsia recomenda o uso de monoterapia sempre que possível. No caso de controle não ter sido obtido com uma das drogas consideradas de primeira linha ou no caso do aparecimento de efeitos colaterais intoleráveis, ela deverá ser substituída por uma outra. Somente quando se esgotarem todas as possibilidades de monoterapia é admissível o uso da politerapia com a combinação racional de duas ou mais drogas maiores ${ }^{16}$. De acordo com o descrito, durante o seguimento, houve a tentativa de instituir monoterapia com o VPA. Caso não houvesse boa resposta, ele seria substituído por drogas consideradas de segunda linha para a condição. Além disso, houve a preocupação em adequar baixas doses procurando aliar eficiência e menor incidência de efeitos colaterais. Dessa forma, após a última consulta, os pacientes foram medicados da seguinte maneira: 20 (76,9\%) em uso do VPA; 3 (11,5\%) em uso do fenobarbital; 2 (7,7\%) em uso de primidona; e $1(3,8 \%)$ com clonazepan em monoterapia associado a controle do ciclo vigíliasono. A dose inicial do VPA preconizada é $10 \mathrm{mg} / \mathrm{Kg} / \mathrm{dia}^{2-5}$ devendo ser aumentada conforme necessidade e tolerância. Em nosso estudo, após seguimento, a dose variou de 500mg/dia a 1500mg/ dia com média de $1000 \mathrm{mg} /$ dia.

\section{REFERÊNCIAS}

1. Wirrel EC, Camfield CS, Camfield PR, Gordon KE, Dooley JM. Long-term prognosis of typical childhood absence epilepsy: remission or progression to juvenile myoclonic epilepsy. Neurology 1996;47:912-918.

2. Delgado-Escueta AV, Enrile-Bacsal F. Juvenile myoclonic epilepsy of Janz. Neurology 1984;34:285-294.

3. Janz D. Epilepsy with impulsive petit mal (juvenile myoclonic epilepsy). Acta Neurol Scand 1985;72:449-459.

4. Yacubian EMT, Grossmann RM, Andrade JCQF, Assis LM. Epilepsia mioclônica juvenil: análise dos aspectos clínicos, eletrencefalográficos e terapêuticos de 36 pacientes. J Liga Bras Epilepsia 1992;5:165-171.

5. Calderón-González R, Ortega F, Ortega-Tamez LC, López-Rivera J, Vallejo-Moreno D, Calderón-Sepúlveda RF. Epilepsia mioclónica juvenil: estudio clínico de 28 pacientes. Rev Mex Pediatr 1988;55:143-144,146-148,150.

6. Serratosa JM, Deltado-Escueta AV, Medina MT, Zhang Q, Iranmanesh R, Sparkes RS. Clinical and genetic analysis of a large pedigree with juvenile myoclonic epilepsy. Ann Neurol 1996;39:187-195.

7. Jain S, Padma MV, Maheshwari MC. Occurrence of only myoclonic jerks in myoclonic epilepsy. Acta Neurol Scand 1997;95:263-267.

8. Durner M, Sander T, Greenberg DA, Johnson K, Beck-Mannagetta G, Janz D. Localization of idiopathic generalized epilepsy on chromosome 6p in families of juvenile myoclonic epilepsy patients. Neurology 1991;41:1651-1655.

9. Greenberg DA, Durner M, Resor S, Rosenbaum D, Shinnar S. The genetics of idiopathic generalized epilepsies of adolescent onset: differences between juvenile myoclonic epilepsy and epilepsy with random grand mal and with awakening grand mal. Neurology 1995;45:942-946.

10. Whitehouse WP, Rees M, Curtis D, et al.. Linkage analysis of idiopathic generalized epilepsy and marker loci on chromosome $6 \mathrm{p}$ in families of patients with juvenile myoclonic epilepsy: no evidence for an epilepsy locus in the HLA region. Am J Hum Genet 1993;53:652-662.

11. Anônimo. Diagnosing juvenile myoclonic epilepsy [Editorial]. Lancet 1992;55:759-760.

12. Grunewald RA, Chroni E, Panayotopoulos CP. Delayed diagnosisof juvenile myoclonid epilepsy. J Neurol Neurosurg Psychiatry 1992;55:497-499.

13. Trevisol-Bittencourt PC, Silva NC, Figueredo R. Neurocisticercose em pacientes internados por epilepsia no hospital regional de Chapecó: região oeste de Santa Catarina. Arq Neuropsiquiatr 1998;56:53-58.

14. Rang HP, Dale MM. Farmacologia. Rio de Janeiro: Guanabara Koogan; 1993.

15. Trevisol-Bittencourt PC, Sander JWAS. Drogas em epilepsia. Arq Cat Med 1989;18:147-155.

16. Sander JWAS, Duncan JS, Trevisol-Bittencourt PC. O tratamento das crises epiléticas. Arq Cat Med 1986;15:77-84. 